\title{
Fabrication of pH-Responsive Nanoparticles with an AIE Feature for Imaging Intracellular Drug Delivery
}

Xing Wang,,$^{\dagger}$ Yanyu Yang, ${ }^{\dagger,+}$ Yaping Zhuang, ${ }^{\dagger,+}$ Peiyuan Gao ${ }^{\dagger+}$ Fei Yang, ${ }^{\dagger,+}$ Hong Shen, ${ }^{\dagger}$ Hongxia Guo ${ }^{\dagger, \star}$ and Decheng $W u^{*, \dagger, *}$

${ }^{\dagger}$ Beijing National Laboratory for Molecular Sciences, State Key Laboratory of Polymer Physics \& Chemistry, Institute of Chemistry, Chinese Academy of Sciences, Beijing 100190, China. E-mail: dcwu@iccas.ac.cn

†niversity of Chinese Academy of Sciences, Beijing 100049, P.R. China 


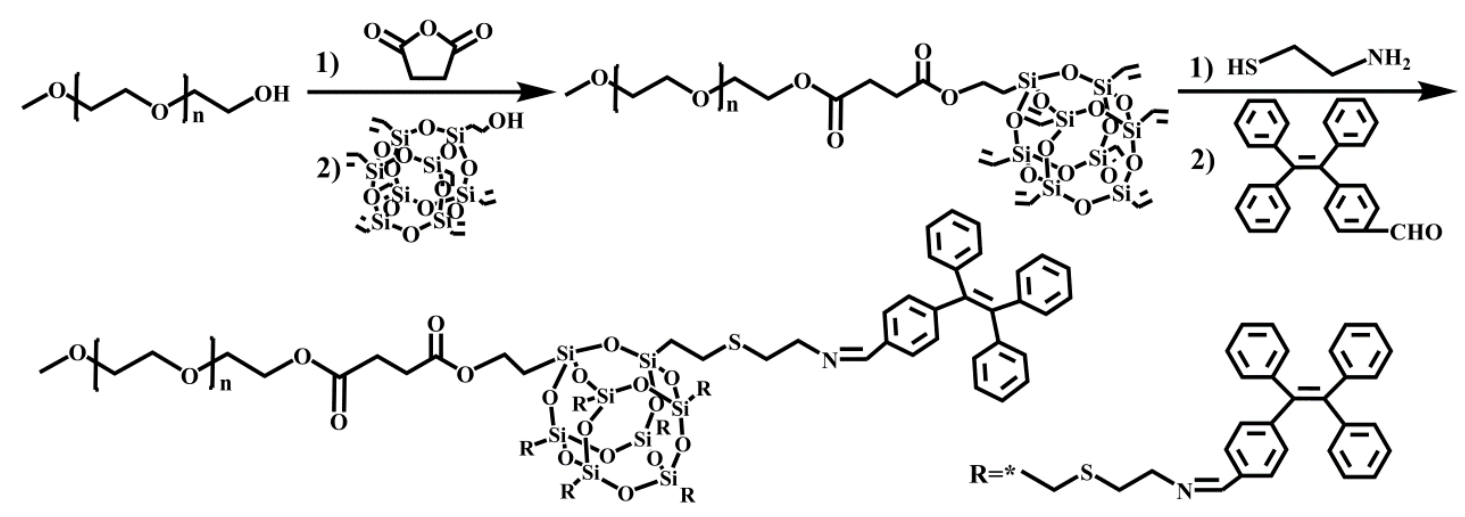

Scheme S1 Synthetic route of the PEG-POSS-(TPE) $)_{7}$ polymer.

DPD Simulation Model and Method. Dissipative particle dynamics (DPD) simulation was performed to understand the assembly of PEG-POSS-(TPE) ${ }_{7}$ in water. In the DPD simulation, we constructed a specific coarse-grained (CG) model of PEG-POSS-(TPE) $)_{7}$ wherein eight branches were attached to one big CG POSS bead. As shown in Scheme S2, there were three types of beads, P, G and T for POSS, PEG and TPE. The atomistic model was coarse-grained according to the molecular segment size in all-atom simulation. In this study, we compared two PEG-POSS-(TPE) $)_{7}$ with different PEG tail length. The ratio of the tail length between the two PEG-POSS-(TPE) $)_{7}$ is six, which is very close to the experiment value 5.42. The interaction parameter $\mathrm{a}_{\mathrm{ij}}$ between different beads was presented in the following Table $\mathrm{S} 1$, which followed our previous work. ${ }^{1}$

Within the CG model, neighboring CG beads are bonded to each other by a harmonic spring potential, $\mathrm{U}_{\mathrm{b}}=0.5 \mathrm{k}_{\mathrm{b}}\left(\mathrm{r}-\mathrm{r}_{0}\right)^{2}$, where $\mathrm{k}_{\mathrm{b}}$ is the spring constant and $\mathrm{r}_{0}$ is the equilibrium bond length. In this study, $\mathrm{k}_{\mathrm{b}}=25$ and $\mathrm{r}_{0}=0.7$. The concentration of PEG-POSS-(TPE $)_{7}$ in the solution $\boldsymbol{\varphi}$ is 0.05 , which is calculated by the following formula. 


$$
\varphi=\frac{N_{P} V_{P}+N_{G} V_{G}+N_{T} V_{T}}{N_{P} V_{P}+N_{G} V_{G}+N_{T} V_{T}+N_{W} V_{W}}
$$

Where $\mathrm{N}_{\mathrm{P}}, \mathrm{N}_{\mathrm{G}}, \mathrm{N}_{\mathrm{T}}$ and $\mathrm{N}_{\mathrm{W}}$ are the number of POSS, PEG, TPE and water beads while $\mathrm{V}_{\mathrm{P}}$, $\mathrm{V}_{\mathrm{G}}, \mathrm{V}_{\mathrm{T}}$ and $\mathrm{V}_{\mathrm{W}}$ are the volume of one bead of POSS, PEG, TPE and solvent, respectively.

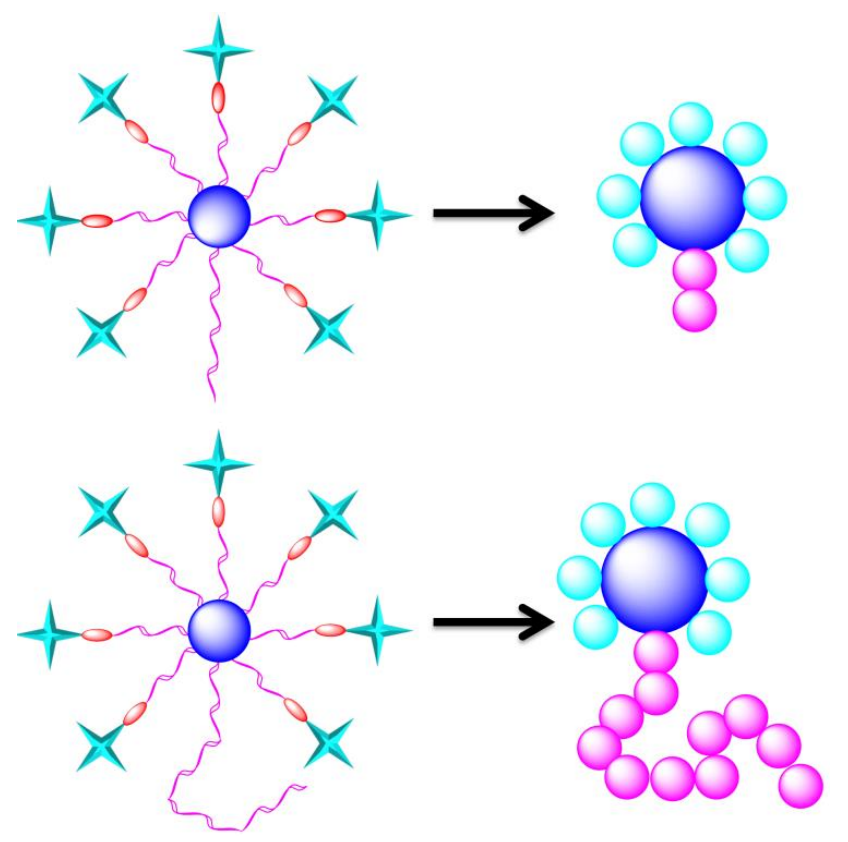

Scheme S2. Scheme of PEG-POSS-(TPE) ${ }_{7}$ CG Model, the Blue, Cyan, and Purple Bead Represent POSS Bead, TPE Bead and PEG Bead, Respectively.

Table S1. The DPD $\mathrm{a}_{\mathrm{ij}}$ Parameters for Different Beads in CG model.

\begin{tabular}{ccccc}
\hline $\mathbf{a}_{\mathbf{i j}}$ & $\mathbf{P}$ & $\mathbf{G}$ & $\mathbf{T}$ & $\mathbf{W}$ \\
\hline $\mathrm{P}$ & 25 & 50 & 25 & 50 \\
$\mathrm{G}$ & & 25 & 25 & 25 \\
$\mathrm{~T}$ & & & 25 & 50 \\
$\mathrm{~W}$ & & & & 25 \\
\hline
\end{tabular}

Here $\mathrm{P}$ for POSS, $\mathrm{G}$ for PEG, T for TPE, $\mathrm{W}$ for water.

The simulations were performed in NVT ensemble with periodical boundary conditions and at a fixed system number density of 3.0. All the CG beads have the same mass as $m=1$. The interaction cutoff radius is set to 1 as the unit of length. The size of POSS is 3 times of 
TPE, PEG or water beads. The temperature is 1.0 . The size of simulation box is $30 \times 30 \times 30$. In the DPD simulations the modified velocity-verlet algorithm was used to integrate the equations of motion with a time step of 0.03 . The simulation systems were pre-equilibrated with $1 \times 10^{6}$ steps by setting $\mathrm{a}_{\mathrm{ij}}=25$ and $\mathrm{T}=1.0$ for all DPD beads. Then we performed another simulation with $3 \times 10^{6}$ steps to observe the morphology evolution of aggregates.

\section{References}

1. Wang, X.; Yang, Y. Y.; Gao, P. Y.; Yang, F.; Shen, H.; Guo, H. X.; Wu, D. C. ACS Macro Lett. 2015, 4, 1321-1326. 


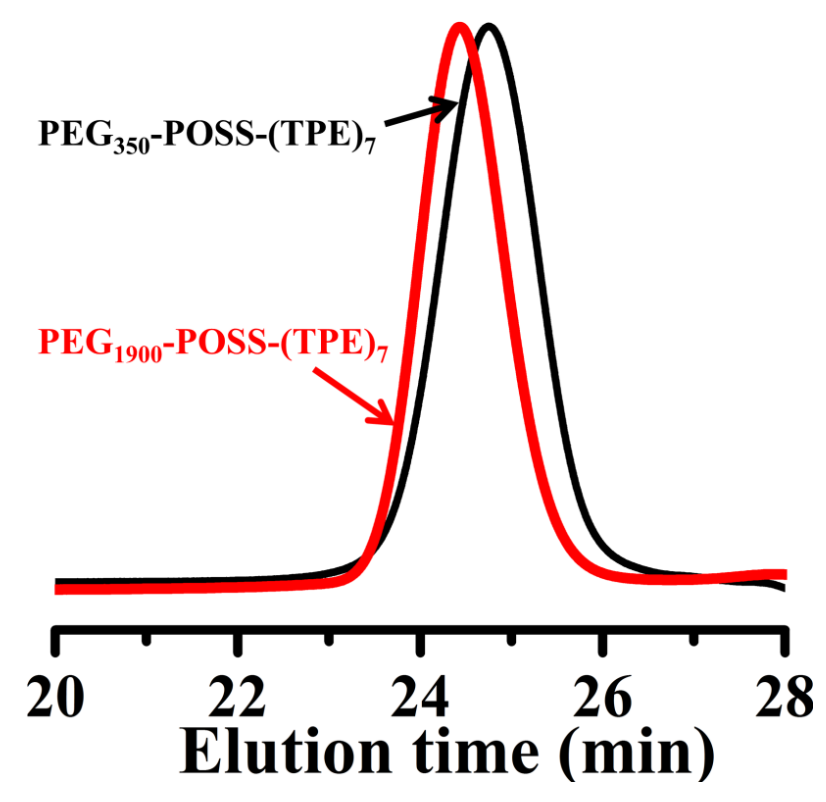

Figure S1. GPC curves of the $\mathrm{PEG}_{350}-\mathrm{POSS}-(\mathrm{TPE})_{7}$ and $\mathrm{PEG}_{1900}-\mathrm{POSS}-(\mathrm{TPE})_{7}$ polymers.

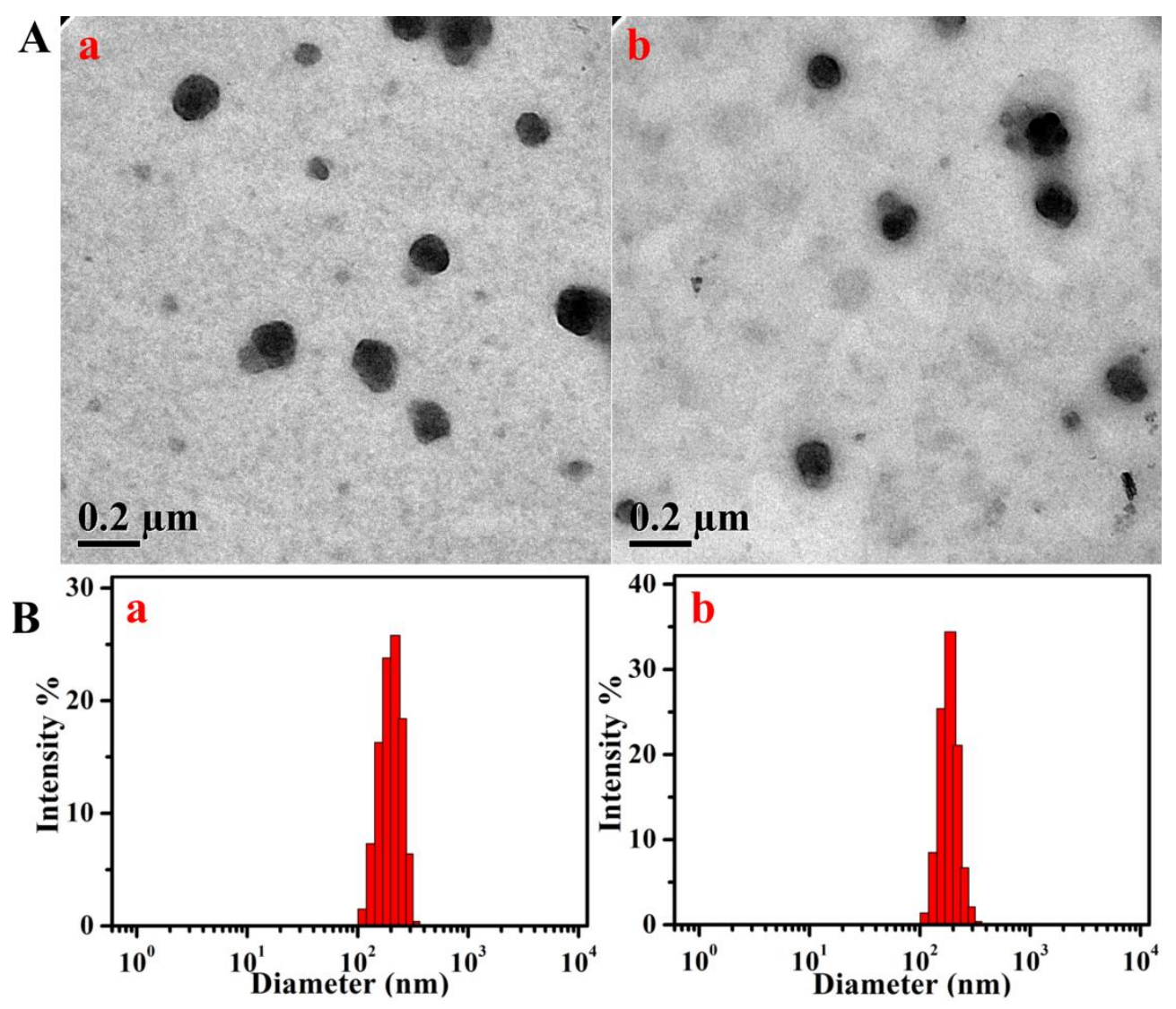

Figure S2. (A) TEM images and (B) DLS profiles of (a) DOX-loaded $\mathrm{PEG}_{350}-\mathrm{POSS}-(\mathrm{TPE})_{7}$ and (b) DOX-loaded PEG $_{1900}$-POSS-(TPE) 7 aggregates. 


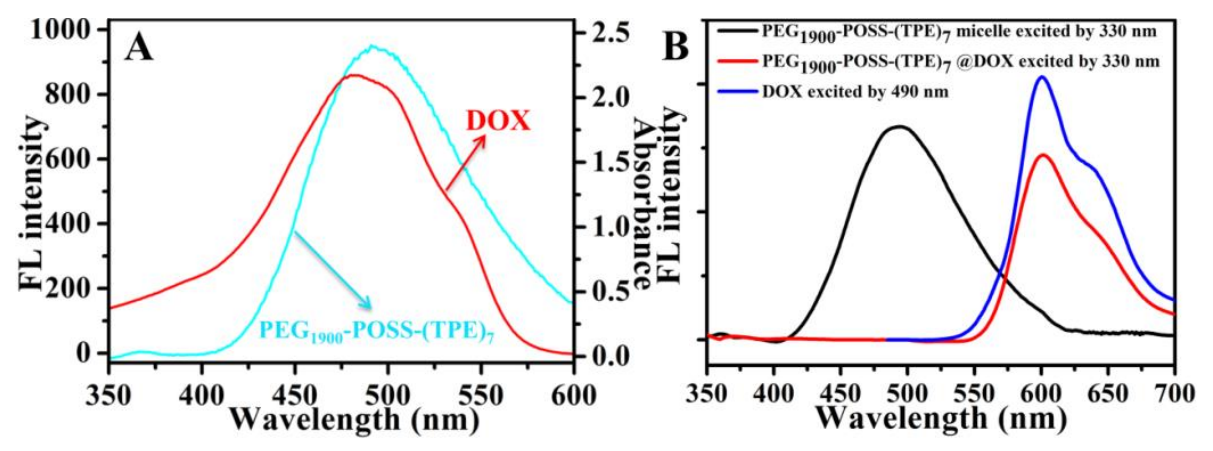

Figure S3. (A) Absorption and emission spectra of $\mathrm{PEG}_{1900}-\mathrm{POSS}-(\mathrm{TPE})_{7}$ (cyan) and DOX (red) couple.

(B) DOX, $\mathrm{PEG}_{1900}-\mathrm{POSS}-(\mathrm{TPE})_{7}$ and DOX-loaded aggregates excited at 330 or $490 \mathrm{~nm}$.

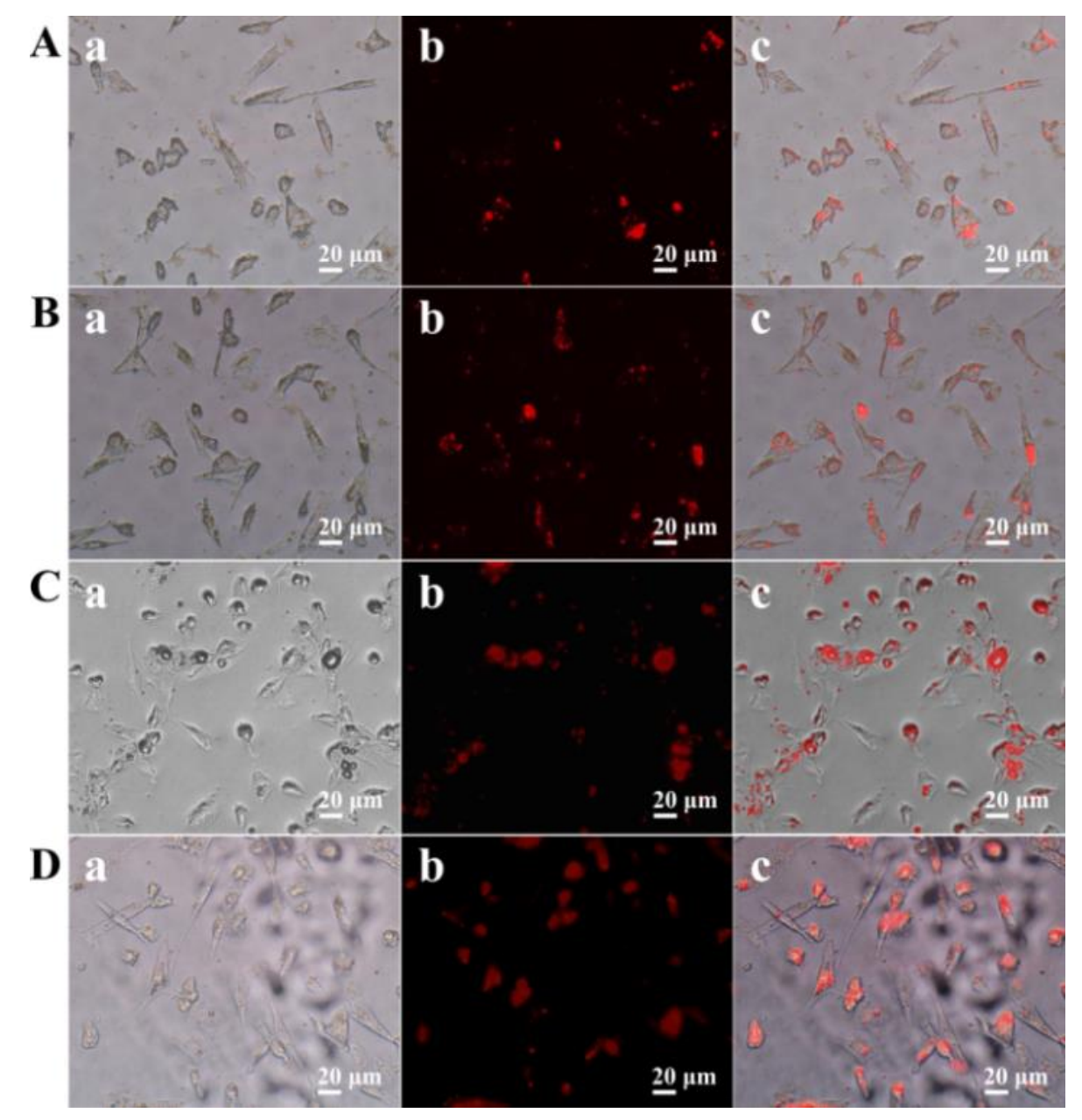

Figure S4. Fluorescent microscope images of MCF-7 cells after incubation with (A, C) DOX-loaded $\mathrm{PEG}_{350}-\mathrm{POSS}-(\mathrm{TPE})_{7}$ and (B, D) DOX-loaded $\mathrm{PEG}_{1900}-\mathrm{POSS}-(\mathrm{TPE})_{7}$ aggregates for 1 and $3 \mathrm{~h}$ with the excitation of $330 \mathrm{~nm}$. a: bright field image, b: red fluorescence image and c: overlap of bright field and fluorescence images. 